\title{
c-Myc shuttled by tumour-derived extracellular vesicles promotes lung bronchial cell proliferation through miR-19b and miR-92a
}

\author{
Cristina Borzi ${ }^{1}$, Linda Calzolari ${ }^{1}$, Anna M. Ferretti ${ }^{2}$, Laura Caleca ${ }^{3}$, Ugo Pastorino ${ }^{4}$, Gabriella Sozzi ${ }^{1}$ and Orazio Fortunato ${ }^{1}$
}

\begin{abstract}
Lung cancer causes approximately one fifth of all cancer deaths. Tumour cells actively communicate with the surrounding microenvironment to support malignant progression. Extracellular vesicles (EVs) play a pivotal role in intercellular communication and modulate recipient cells by delivering their contents, including proteins and nucleic acids such as microRNAs (miRNAs). We isolated EVs from the conditioned medium (CM) of human lung cancer cell lines and plasma of lung cancer patients and cancer-free smokers using an ultracentrifugation method. A significant increase in bronchial HBEC-KRAS ${ }^{\text {V12high }}$ cell proliferation, confirmed by cell cycle analysis, was observed after treatment with cancer-derived EVs. Lung cancer-derived EVs induced transcription of the pri-miR-92a gene, resulting in the overexpression of mature miR-19b and miR-92a in recipient bronchial cells. Modulation of these two miRNAs using miRNA mimics or inhibitors confirmed their ability to promote proliferation. In silico analysis and experimental validation showed that miR-19b and miR-92a impaired the TGF-beta (TGFB) pathway and identified TGFBRI and TGFBRII as target genes involved in EV-mediated bronchial cell proliferation. Interestingly, the oncoprotein c-Myc, a well-known miR-17-92 cluster activator, was detected only in the EVs derived from lung cancer patients and cell lines and was able to modulate the proliferation of HBEC-KRAS ${ }^{12 h i g h}$ recipient cells. These data support the role of c-Myc shuttling in lung cancer-derived EVs in inducing the upregulation of onco-miR-19b and miR-92a expression with concomitant impairment of the TGFB signalling pathway in recipient cells.
\end{abstract}

\section{Introduction}

Lung cancer is one of the most common and malignant types of cancer worldwide ${ }^{1,2}$. In 2018, it was estimated that lung cancer accounted for $14 \%$ of all new diagnoses and $\sim 25 \%$ of all cancer deaths ${ }^{3}$. The high mortality is due to the absence of symptoms in early stages ${ }^{4}$ and the lack of effective therapeutic interventions, despite improvements achieved in recent years with immunotherapy ${ }^{2,5}$. Therefore, to improve survival, there is still a clinical need

\footnotetext{
Correspondence: Orazio Fortunato (orazio.fortunato@istitutotumori.mi.it) ${ }^{1}$ Tumor Genomics Unit, Department of Research, Fondazione IRCCS Istituto Nazionale dei Tumori, 20133 Milan, Italy

${ }^{2}$ Lab. Di nanotecnologie, Istituto di scienze e tecnologie Molecolari CNR, 20138 Milan, Italy

Full list of author information is available at the end of the article. These authors contributed equally: Gabriella Sozzi, Orazio Fortunato Edited by C. Munoz-Pinedo
}

to investigate the molecular mechanisms underlying lung cancer development and progression. Lung tumourigenesis is strongly regulated by the complex interplay between tumour and stromal cells, including fibroblasts ${ }^{6}$, endothelial cells ${ }^{7}$ and immune cells ${ }^{5,8}$. Interactions among cells in the tumour microenvironment are mediated by several mechanisms: cell-cell contact (receptor-mediated interactions and gap junctions) and paracrine signals (growth factors, cytokines and chemokines) as well as by extracellular vesicles $(\mathrm{EVs})^{9,10}$.

EVs are spherical, bilayered, membranous vesicles generated by all cell types in mammalian organisms. EVs are generally recognised as exosomes $(30-150 \mathrm{~nm})$, microvesicles $(0.1-1 \mu \mathrm{m})$ and apoptotic bodies $(0.8-5 \mu \mathrm{m})$. EVs are constitutively released by different cell types to mediate cell-to-cell communication both in

\section{(c) The Author(s) 2019}

(c) (i) Open Access This article is licensed under a Creative Commons Attribution 4.0 International License, which permits use, sharing, adaptation, distribution and reproduction in any medium or format, as long as you give appropriate credit to the original author(s) and the source, provide a link to the Creative Commons license, and indicate if changes were made. The images or other third party material in this article are included in the article's Creative Commons license, unless indicated otherwise in a credit line to the material. If material is not included in the article's Creative Commons license and your intended use is not permitted by statutory regulation or exceeds the permitted use, you will need to obtain permission directly from the copyright holder. To view a copy of this license, visit http://creativecommons.org/licenses/by/4.0/. 
normal and pathological states ${ }^{11}$. EVs contain a heterogeneous composition of biomolecules, including lipids, proteins and nucleic acids such as DNA and RNA (mRNAs, long non-coding RNAs, microRNAs (miRNAs) $)^{11-13}$. Cells can use EVs to shuttle biomolecules to neighbouring or distant cells and influence recipient cell functionality ${ }^{11,14}$. Recent evidence shows that EVs, in particular exosomes, are closely related to lung carcinogenesis ${ }^{15}$. Tumour-derived exosomes play a crucial role in the growth and progression of lung cancer by modulating tumour angiogenesis ${ }^{16}$ and epithelial-to-mesenchymal transition (EMT) ${ }^{17}$. In addition, Lobb RJ et al. demonstrated that exosomes derived from oncogenically transformed bronchial epithelial cells transferred chemoresistance to recipient cells through exosomal ZEB1 mRNA transfer ${ }^{18}$.

MiRNAs represent a class of small non-coding RNAs that act as master regulators of gene expression ${ }^{19}$. In cancer, they can act as tumour suppressors or oncogenes $^{20}$. We previously generated a miRNA risk classifier based on the reciprocal ratios of 24 plasma-derivedmiRNAs associated with lung cancer development and prognosis $^{21,22}$. We also demonstrated the functional roles of specific miRNAs composing the signature (miR-486 and miR-660) in lung tumourigenesis ${ }^{23,24}$, supporting their central roles in the modulation of cancer-associated pathways, such as the p53. An interesting work showed that miR-21 and miR-29a contained in lung tumourderived exosomes were able to bind Toll-like receptors (TLRs) expressed by immune cells, leading to a TLRmediated inflammatory response that supported lung tumour growth and metastasis ${ }^{25}$. It has been shown that exosomal miR-23a, which is overexpressed in hypoxic lung cancer, enhances angiogenesis and vascular permeability through its targets prolyl hydroxylase (PHD) and tight junction protein- $1^{26}$. The same authors identified miR-103a contained in hypoxic lung cancer-derived exosomes as a mediator of M2-phenotype polarisation in macrophages through a PTEN-dependent mechanism ${ }^{27}$.

To date, the functional roles of miRNAs associated with EVs in lung cancer development and progression are largely unknown. The present study aimed to characterise EVs derived from lung cancer cells and patients and elucidate their pro-tumourigenic roles in the lung epithelium, focusing on the functional interactions between EVs and miRNAs. Lung cancer is a multistep process resulting in specific oncogene and/or tumour suppressor gene alterations in epithelial cells due to a prolonged smoke exposure. To this purpose, we utilised nontumorigenic Bronchial Epithelial HBEC-KRAS ${ }^{\mathrm{V} 12 \mathrm{high}}$ as recipient cells. These cells are oncogenically-modified $\mathrm{HBECs}^{28}$ that show a certain degree of 'plasticity' versus a partial malignant transformation. These features make HBECs-KRAS ${ }^{\text {V12high }}$ an appropriate model to investigate the effects of tumour-derived EVs in neighbouring cells and their contribution to lung carcinogenesis.

\section{Results \\ Isolation and characterisation of EVs from lung cancer cell lines}

EVs from the lung cancer cell lines A549 and LT73 and HBEC-KRAS ${ }^{\text {V12high }}$ were isolated from conditioned medium $(\mathrm{CM})$ and characterised. Size distribution analysis using NanoSight identified a major peak between 50 and $200 \mathrm{~nm}$, with modes of 89 and $96 \mathrm{~nm}$ for A549- and LT73-derived EVs (EVs-A549 and EVs-LT73), respectively (Fig. 1a and Table 1). A similar size distribution was observed for EVs derived from HBEC-KRAS ${ }^{\text {V12high }}$ cells, with a diameter's mode about $100 \mathrm{~nm}$ (Supplementary Fig. 1a and Table 1). Notably, we observed a greater amount of EVs, approximately four times higher, being released by tumour cells than by HBEC-KRAS ${ }^{\text {V12high }}$ cells, as measured by their total protein content (Supplementary Fig. $1 \mathrm{~b}$ and Table 1 ).

The results obtained by NanoSight were confirmed by transmission electron microscopy (TEM) analysis. The vesicles showed a spherical shape with a relatively wide size distribution (Fig. 1b, Supplementary Fig. 1c, d). EVsA549 had a mean diameter $\left(d_{\mathrm{m}}\right)$ of $56.7 \mathrm{~nm}$, whereas a larger diameter, $d_{\mathrm{m}}=69.8 \mathrm{~nm}$, was detected for EVsLT73. Instead, HBEC-KRAS ${ }^{\text {V12high }}$ derived EVs showed the smallest average diameter $\left(d_{\mathrm{m}}=38.2\right)$ (Supplementary Fig. 1d).

FACS and WB analysis revealed high levels of the exosome-enriched proteins CD9, CD63, CD81 and Alix (Fig. 1c, d, Supplementary Fig. 1e). To explore the protumourigenic potential of lung cancer-derived EVs, we evaluated the in vitro interaction between tumoural EVs and HBEC-KRAS ${ }^{\text {V12high }}$, mimicking a possible in vivo interplay between a tumour and the surrounding epithelial component. To this end, we treated HBECKRAS $^{\text {V12high }}$ cells with PKH67-labelled EVs. After 24h of culture, confocal laser scanning microscopy images showed the uptake of EVs-A549 and EVs-LT73 by recipient cells (Fig. 1e). FACS analysis confirmed the presence of EVs inside cells at different time points $(24,48$ and 72 h) (Supplementary Fig. 2).

\section{Lung cancer-derived EVs promote bronchial epithelial cell proliferation through miR-19b and miR-92a}

To explore the interaction between tumoural EVs and HBEC-KRAS ${ }^{\text {V12high }}$, we treated HBEC-KRAS ${ }^{\text {V12high }}$ cells with EVs $(15 \mu \mathrm{g})$ and the corresponding CM-EVs depleted. Untreated cells were used as an additional control. Compared to EV-depleted CM treated and untreated cells, EV-treated HBEC-KRAS ${ }^{\text {V12high }}$ cells showed a significant increase in proliferation at $72 \mathrm{~h}$ (Fig. 2a). This result was confirmed by carboxyfluorescein succinimidyl 


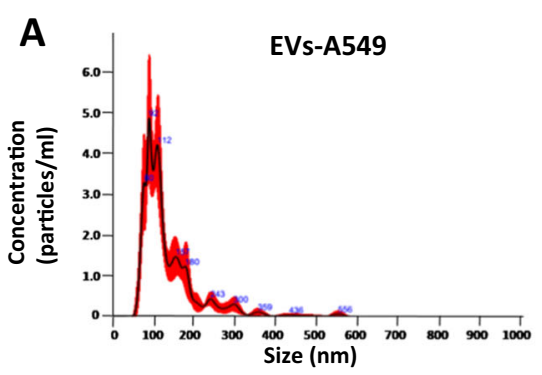

B
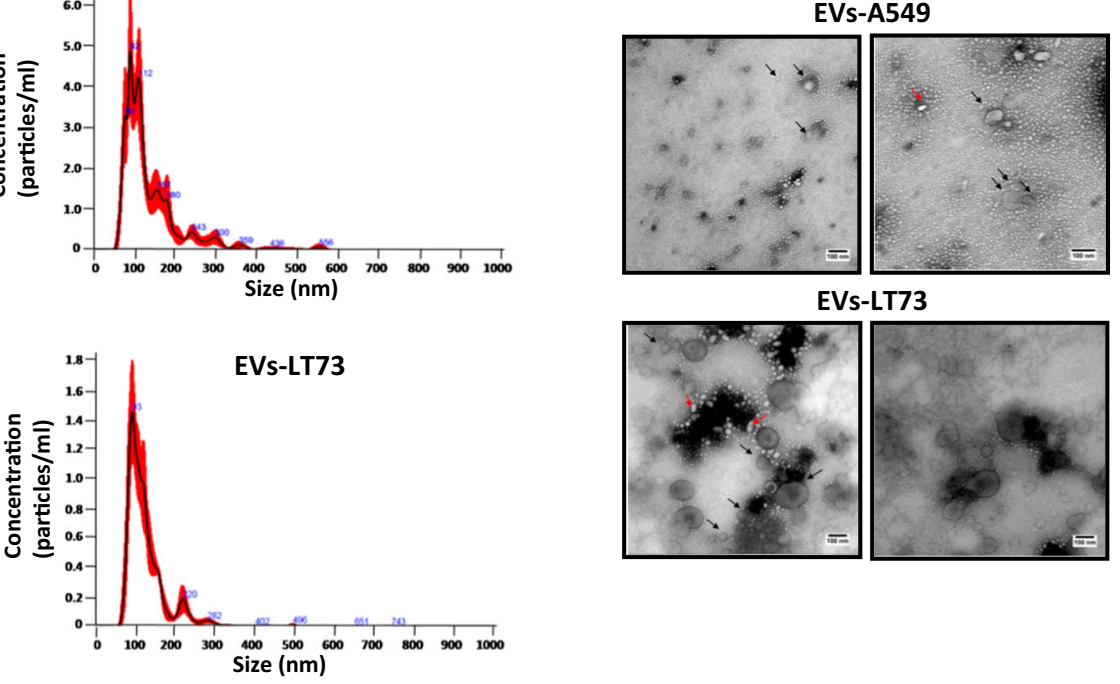

EVs-LT73

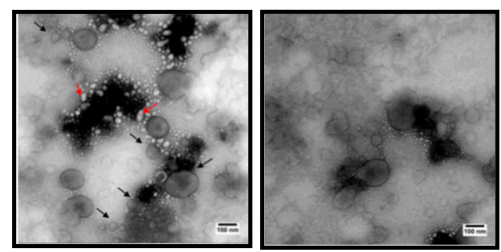

C

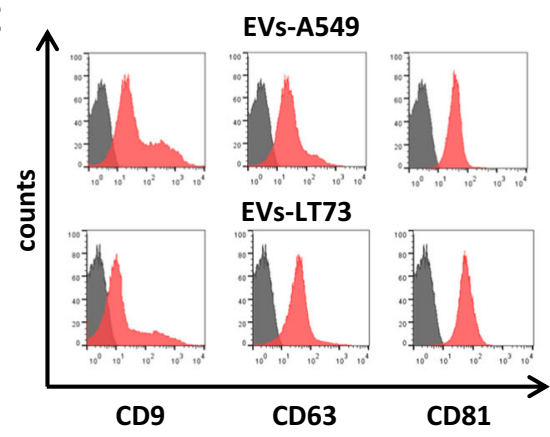

E
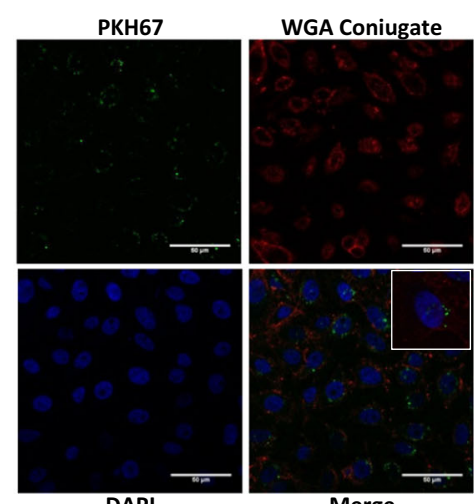

WGA Coniugate

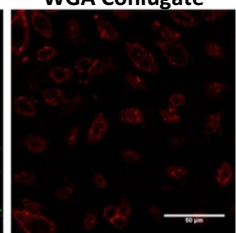

D
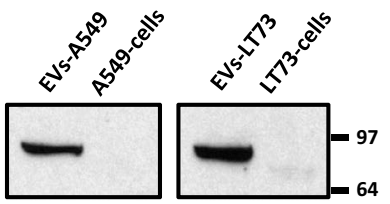

CD9
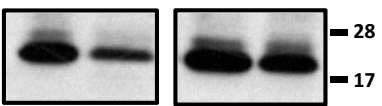

CD81
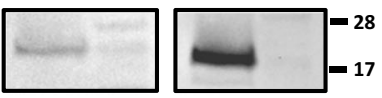

EVs-LT73

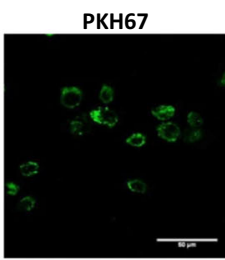

WGA Coniugate

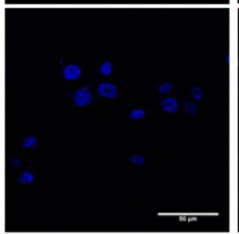

DAPI

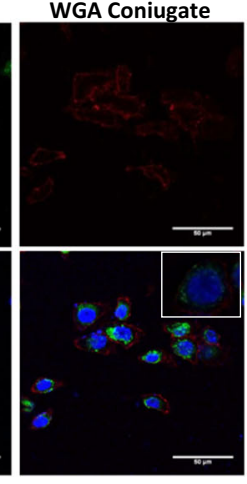

Merge

Fig. 1 Characterisation of EVs isolated from lung cancer cell lines. a Size distribution and concentration of A549 cell- and LT73 cell-derived EVs obtained using the NanoSight Instrument. b Representative images of EV pellets observed by TEM. Black arrows indicate EVs, while red arrows represent artefacts. c, $\mathbf{d}$ Analysis of the exosome-enriched proteins CD9, CD63, CD81 and Alix by FACS (c, representative images, $n=3$ ) and western blotting (d). Cellular lysates were used as a control for western blot analysis. e Representative confocal laser scanning microscopy images of PKH67labelled EV uptake by 633-WGA-labelled HBEC-KRASV12high recipient cells after $24 \mathrm{~h}$. Green $=$ PKH67-labelled EVs, red $=$ WGA-conjugated recipient cells and blue $=$ nuclei 
Table 1 Size and concentration of EVs isolated from different lung cell lines

\begin{tabular}{llll}
\hline & EVs-A549 & EVs-LT73 & EVs-HBEC-KRAS ${ }^{\text {V12high }}$ \\
\hline Mean size $(\mathrm{nm})$ & $143.9 \pm 8.7$ & $130.3 \pm 6.3$ & $121.9 \pm 6.3$ \\
Mode size $(\mathrm{nm})$ & $89.3 \pm 7.3$ & $96.2 \pm 4.5$ & $99.6 \pm 7.2$ \\
Particles/ml & $3.6 \times 10^{10} \pm 2.5 \times 10^{9}$ & $8.9 \times 10^{10} \pm 3.9 \times 10^{9}$ & $9.0 \times 10^{9} \pm 9.6 \times 10^{8}$ \\
\hline
\end{tabular}

NanoSight analysis was performed using $5 \mu \mathrm{g}$ of EVs from each cell type.

ester (CFSE) analysis (Fig. 2a, Supplementary Fig. 3). Moreover, we tested the capability of tumoural EVs to support 3D cell culture. Treatment with EVs-A549 and EVs-LT73 resulted in enhanced 3D proliferation (defined as a cluster of at least 10 cells), as compared to control cells over time (Fig. 2b). In addition, cell cycle analysis revealed a significant increase of cells in the G2/M phase in EV-treated cells compared to controls (Fig. 2c).

To characterise the pro-tumourigenic effects of EVs, we evaluated migration and EMT process in HBECKRAS $^{\text {V12high }}$ cells after EV treatment. A wound healing assay revealed no difference in wound closure time between the EVs-A549- or EVs-LT73-treated cells and the corresponding controls (Supplementary Fig. 4a). Gene expression analysis did not show significant modulation of EMT-related gene levels (Supplementary Fig. 4b), indicating no alterations in either migratory ability or epithelial phenotype occurred. These results agree with published data, which showed that these cells need to grow in serum-supplemented medium to undergo $\mathrm{EMT}^{28}$.

To investigate the involvement of EV-miRNAs in the enhancement of HBEC-KRAS ${ }^{\mathrm{V} 12 \mathrm{high}}$ proliferation, we analysed miRNA expression in recipient cells, focusing on 24 miRNAs that we previously implicated in the pathogenesis and aggressiveness of lung tumours ${ }^{21}$. Among the 24 miRNAs, only miR-19b and miR-92a exhibited significant upregulation in recipient cells treated with EVs derived from both cell lines compared to control (Fig. 2d, e, Supplementary Fig. 5). To understand whether EVshuttled miR-19b and miR-92a could be directly transferred to recipient cells, we evaluated the expression of pri-miR-92a (a precursor of both miRNAs) in treated cells. We observed a statistically significant increase in pri-miR-92a expression in the EV-treated cells as shown in Fig. 2f. Since pri-miR-92a was not detected inside EVsA549 and EVs-LT73 (data not shown), these results suggest endogenous transcription of pri-miR-92a and, consequently, of the two miRNAs. To exclude miRNAs transfer, we isolated EVs from A549 where miR-19b and miR-92a were silenced by LNA inhibitors, obtaining EVs devoided of the two miRNA (EVs-A549(LNA)) (about 95 and $90 \%$ of reduction for $\mathrm{miR}-19 \mathrm{~b}$ and $\mathrm{miR}-92 \mathrm{a}$, respectively) (Supplementary Fig. 6a). HBEC-KRAS ${ }^{\text {V12high }}$ exposed to EVs-A549(LNA) showed pro-tumorigenic phenotype as observed for WT-EVs: increase in cell growth and G2/M cell cycle phase (Supplementary Fig. 6b). Upregulation of the two miRNAs was observed in recipient cells upon EVs-A549(LNA) exposure, excluding an EVsmediated miRNAs transfer (Supplementary Fig. 6c).

The roles of miR-19b and miR-92a on cell proliferation were also investigated using miRNA mimics $(5 \mathrm{nM})$ in HBEC-KRAS ${ }^{\text {V12high }}$ cells, and 20 and $28 \%$ increases in cell numbers following miR-19b (mim-19b) or miR-92a (mim-92a) overexpression, respectively, compared to scramble (scr) were observed ( $p<0.01$; Fig. 3a, left). This result was confirmed by CFSE analysis (Fig. 3a, middle) and cell cycle analysis (Fig. 3a, right), where an increase in the proportion of cells in the G2/M phase was detected. miR-19b and miR-92a levels following miRNAs mimics treatment are shown in Supplementary Fig. 7a. Of note, overexpression of the two miRNAs was comparable to those induced by EVs exposure (Supplementary Fig. 7b).

To confirm the crucial roles of these two miRNAs in the aforementioned phenotypic modulation, we performed miR-19b and miR-92a inhibition in recipient cells using LNA inhibitors and then evaluated HBEC-KRAS V12high cell proliferation after EV treatment (Fig. 3b-d, Supplementary Fig. 8). Compared to no treatment, treatment with LNA-19b or LNA-92a caused 90 and 95\% reductions in miR-19b and miR-92a expression levels, respectively. Treatment with EVs or EV-depleted CM did not enhance miRNA expression in LNA-treated cells (Supplementary Fig. 8). Accordingly, LNA treatment reversed the advantages conferred by EVs in cell viability (Fig. 3b) and CFSE analyses (Fig. 3c). In fact, inhibition of the miRNAs reduced cell viability and differences in the cell cycle were no longer detected (Fig. 3b-d).

To verify the specificity of the functional effects observed after tumour cell-derived EV exposure, we repeated the experiment using EVs derived from nontumourigenic HBEC-KRAS ${ }^{\text {V12high }}$ cells (EVs- HBEC$\left.\mathrm{KRAS}^{\mathrm{V} 12 \mathrm{high}}\right)$. Notably, the proliferation of the recipient cells was not affected by exposure to EVs- HBECKRAS $^{\text {V12high }}$ (Supplementary Fig. 9a-c).

Overall, these results demonstrated the ability of lung cancer EVs to induce the upregulation of miR-19b and miR-92a expression and increase the proliferation of recipient epithelial cells. 


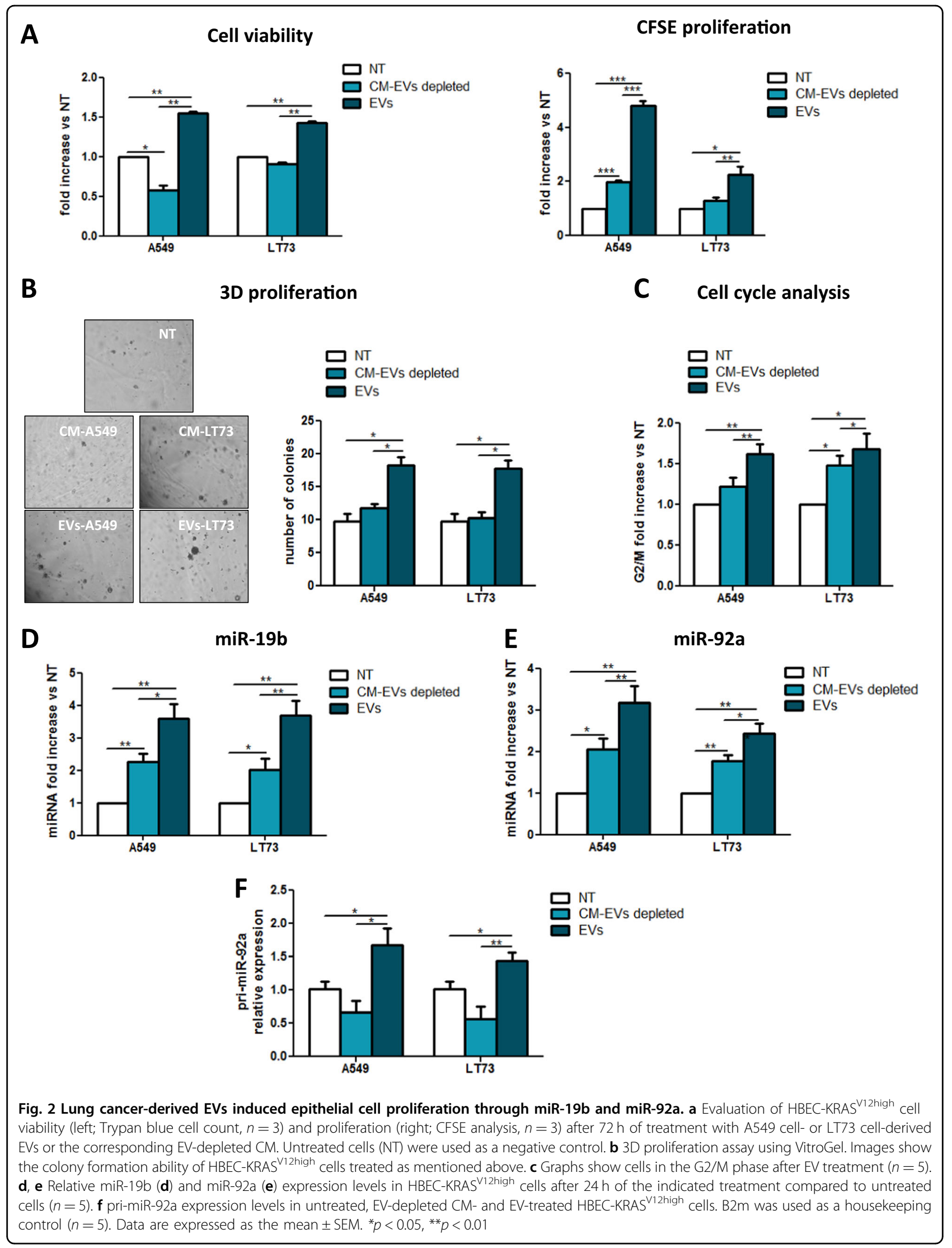




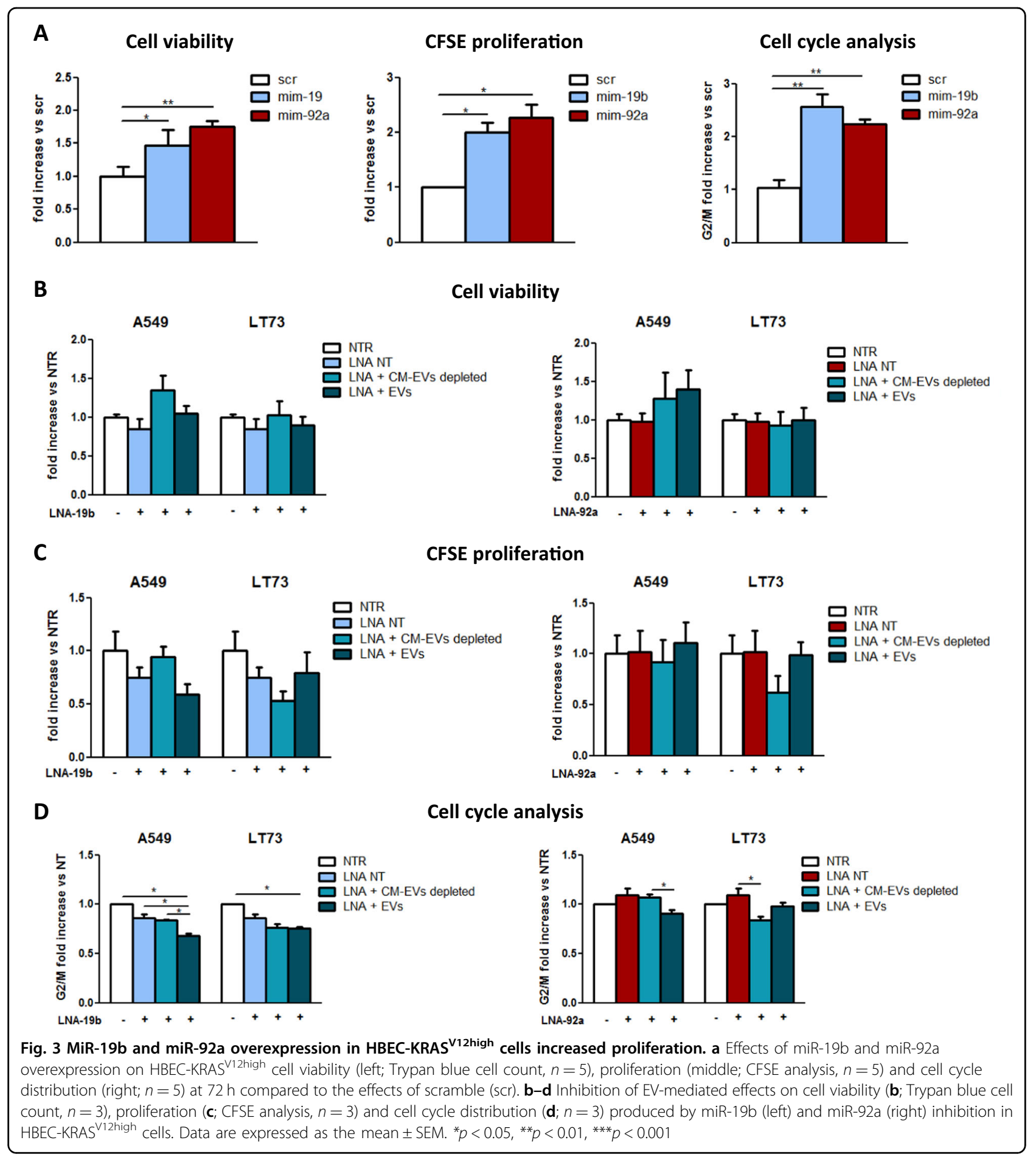

TGFBRs are involved in EV-mediated epithelial cell proliferation

In silico analyses of experimentally validated miR-19b and miR-92a targets were performed to understand the molecular mechanisms underlying HBEC-KRAS ${ }^{\mathrm{V} 12 \text { high }}$ cell proliferation. KEGG pathway analysis revealed associations of miR-19b and miR-92a target genes with cancer pathways, including NSCLC-related pathways, cell cyclerelated pathways and TGF-beta (TGFB) signalling, as illustrated in Fig. 4a. Several studies have reported downregulation of TGFB signalling after miR-17-92 overexpression in different types of cancer, including lung cancer $^{29-31}$. Therefore, we focused on the genes involved in TGFB signalling, and target prediction revealed 


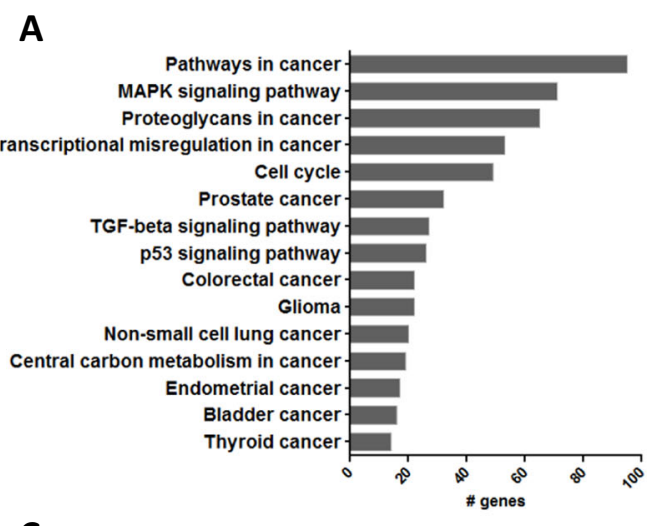

C

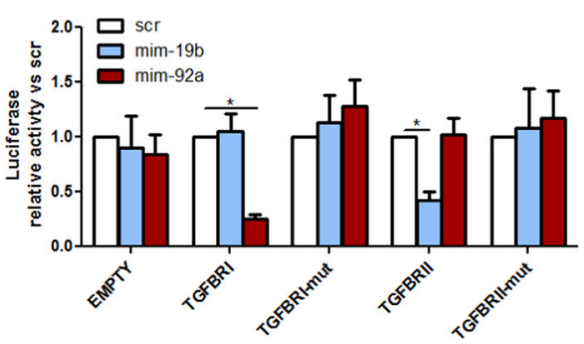

D

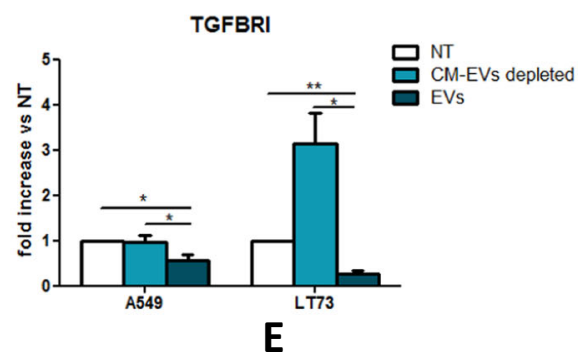

B

$\begin{array}{ll}\text { TGFBRI: } & \text { 5' GAGGAACAUAAUUCAUGCAAUU 3' } \\ \text { miR-92a-3p: } & \text { 3' UGUCCGGCCCUGUUCACGUUAU 5' } \\ \text { TGFBRI-Mut: } & \text { 5' I I I I I } \\ & \\ \text { TGFBRII: } & \text { 5' CAAUAGCCAAUAACAUUUGCACU 3' } \\ \text { miR-19b-3p: } & \text { 3' AGUCAAAACGUACCUAAACGUGU 5' } \\ \text { TGFBRII-Mut: } & \text { 5' I I I I I I I }\end{array}$

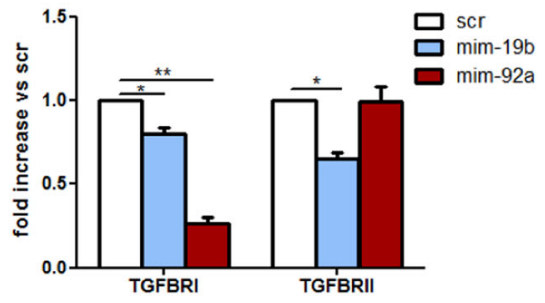

$\mathbf{F}$
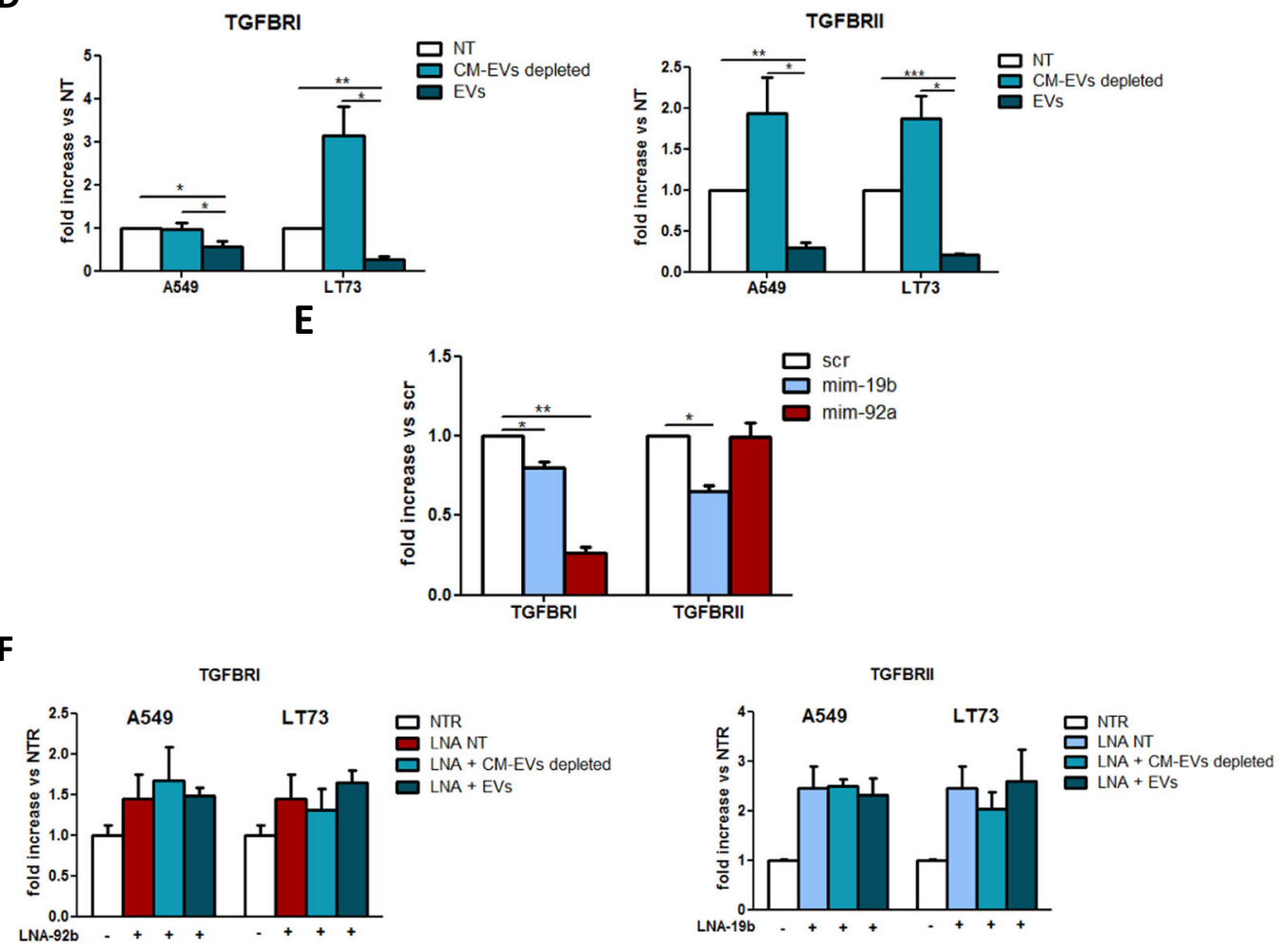

Fig. 4 TGFBRs were involved in EV-mediated proliferation. a Gene enrichment analysis of experimentally validated miR-19b and miR-92a target genes. Only cancer-related pathways are shown in the graph. $\mathbf{b}$ miR-92a and miR-19b seed sequence alignments with the $3^{\prime}$ UTRs of TGFBRI and TGFBRII, respectively. Both wild-type and mutated sequences of TGFBRs $3^{\prime} U T R$ are shown (mutated sequences are indicated in red). c Bar graph showing relative luciferase activity, compared to control, for wild-type and mutated TGFBRI- and TGFBRII- $3^{\prime} U$ TR reporters. WT and EMPTY control plasmids were transfected into HEK293 cells alone or with mim-19b, mim-92a or scr-control. Reporter activity was measured after $48 \mathrm{~h}$ and normalised to Renilla luciferase activity $(n=3)$. d Modulation of TGFBRI and TGFBRIl protein expression in HBEC-KRAS ${ }^{\text {V12high }}$ cells after $72 \mathrm{~h}$ of treatment with EVs or EV-depleted CM $(n=3)$. e Modulation of TGFBRI and TGFBRII protein expression following miR-19b and miR-92a overexpression ( $n=3)$. $\mathbf{f}$ Effects of miR-92a (left) and miR-19b (right) inhibition on TGFBRI and TGFBRIl protein expression, respectively, after the indicated treatments $(n=3)$. Data are expressed as the mean \pm SEM. ${ }^{*} p<0.05,{ }^{* *} p<0.01,{ }^{* * *} p<0.001$ 
TGFBRI and TGFBRII as potential miR-92a and miR-19b targets, respectively (Fig. 4b). To confirm that TGFBRs are direct targets of miR-19b and miR-92a, we performed a luciferase reporter assay and observed downmodulation of luciferase activity when HEK-293 cells were cotransfected with miRNA mimics (75 and $58 \%$ of reduction for TGFBRI-mim-92a and TGFBRII-mim19b, respectively) (Fig. 4c). Target specificity was validated using a $3^{\prime}$ UTR EMPTY and mutated 3'UTR-TGFBRs vectors, as depicted in Fig. $4 \mathrm{~b}$, where no changes in luciferase activity were detected (Fig. 4c). Therefore, TGFBRI and TGFBRII were experimentally validated as miR-92a and miR-19b targets, respectively, in HBECKRAS $^{\text {V12high }}$ cells.

Using flow cytometry analysis, we found that the expression of TGFBRI and TGFBRII was downregulated in HBEC-KRAS ${ }^{\text {V12high }}$ cells treated with cancer-derived EVs compared to untreated cells (50 and 61\% reductions in TGFBRI expression and 44 and $79 \%$ reductions in TGFBRII expression for EVs-A549 and EVs-LT73, respectively, p < 0.05 ) and CM control-treated cells (44 and $73 \%$ reductions in TGFBRI expression and 69 and 79\% reductions in TGFBRII expression for EVs-A549 and EVs-LT73, respectively, $p<0.05$ ) (Fig. $4 \mathrm{~d}$ and Supplementary Figs. 10a-12). A similar result was observed also using EVs depleted of miR19b and miR-92a (Supplementary Fig. 6d).

We also observed downmodulation of the respective targets, both at the mRNA (Supplementary Fig. 10b) and protein levels (Fig. 4e, Supplementary Figs. 11 and 12), after miR-19b and miR-92a overexpression. Compared to no treatment, inhibition of miR-19b or miR-92a in HBECs increased the protein level of TGFBRII or TGFBRI, respectively (Fig. 4f). Notably, LNA-92a (Fig. 4f, left) and LNA-19b (Fig. 4f, right) prevented TGFBR downmodulation in EV-treated cells. TGFBRI and TGFBRII expression levels in recipient cells were not affected by EVsHBEC-KRAS ${ }^{\text {12high }}$ exposure. This is in agreement with the unchanged pri-miR-92a expression level (Supplementary Fig. 9d, e). An impairment of TGFB pathway after EVs exposure was observed through the modulation of downstream targets such as CDC25A, E2F-1, p15, p21, and p57, as already described ${ }^{32-35}$ (Supplementary Fig. 10c).

These data indicate that modulation by miR-19b and miR-92a impairs the TGFBR pathway in bronchial epithelial cells, contributing to the increase in the proliferative rate induced by lung cancer-derived EVs.

\section{c-Myc shuttled by tumour-derived EVs promotes malignant transformation of epithelial recipient cells}

To better define the molecular mechanism linking EV treatment to epithelial cell proliferation, we searched for the presence of a transcriptional activator of pri-miR-92a in the EV cargo. The oncoprotein c-Myc is a well-known miR-17-92 cluster activator ${ }^{36}$. Therefore, we tested EVs-
A549 and EVs-LT73 for the presence of c-Myc by flow cytometry, and we observed that both samples were positive, showing $\sim 10$ and $5 \% \mathrm{c}-\mathrm{Myc}^{+}$-EVs among the EVs-A549 and EVs-LT73, respectively (Fig. 5a, left and Supplementary Fig. 13a).

EVs-A549(LNA) displayed same levels of c-Myc expression compared to A549 wild-type EVs (Supplementary Figs. 6e and 13a). C-Myc protein was detected by WB in A549 and LT73 cells and at lower levels in HBECKRAS $^{\text {V12high }}$ (Supplementary Fig. 13b). Moreover, c-Myc intracellular expression level and EVs cargo were quantified by enzyme-linked immunosorbent assay (ELISA) (c -Myc: about 1.1 and $1.4 \mathrm{ng} / \mu \mathrm{l}$ for A549 and LT73 cellular lysates, Supplementary Fig. 13c, and 58 and $30 \mathrm{pg} / \mu \mathrm{l}$ for EVs-A549 and EVs-LT73 respectively, Fig. 5a, right), showing that about 4 and $2 \%$ of cellular c-Myc was released within EVs by A549 and LT73, respectively. In addition, EVs-HBEC-KRAS V12high had lower amount of cMyc compared to tumour EVs (Fig. 5a, Supplementary Fig. 13a). To prove c-Myc-shuttling, we measured c-Myc level in HBEC-KRAS ${ }^{\text {V12high }}$ after EVs exposure observing a greater increase of c-Myc in both EVs-A549 and EVsLT73 treated cells compared to controls (Fig. 5b). We detected modulation of miRNAs known to be regulated by c-Myc ${ }^{36}$ such as miR-9, miR-17, miR-18a, miR-34a, let7b (Supplementary Fig. 13d). These data suggest an EVsmediated transfer of $\mathrm{c}-\mathrm{Myc}$ from lung tumour to epithelial cells.

To prove that EV-shuttled c-Myc was responsible for the phenotypic changes observed, we transiently transfected c-Myc plasmid into HBEC-KRAS ${ }^{\text {V12high }}$ cells. A significant increase in cell proliferation was observed by both Trypan blue and CFSE analysis (Fig. 5c, Supplementary Fig. 14), an increase in the number of colonies (Fig. 5d), and an enhancement in G2/M phase was noted (Fig. 5e). Overexpression of c-Myc induced pri-miR-92a transcription followed by miR-19b and miR-92a upregulation (Fig. 5f-g). We detected 50\% reductions in TGFBRI- and TGFBRII-positive cell numbers in c-Mycoverexpressing cells compared to control (Fig. 5h).

These results suggest a mechanistic explanation for the ability of lung cancer-derived EVs to induce a protumourigenic feature in HBEC-KRAS ${ }^{\mathrm{V} 12 \text { high }}$ cells.

\section{Lung cancer patient-derived EVs enhance bronchial epithelial cells proliferation through EVs shuttled c-Myc -TGFB pathway interplay}

To verify whether our proposed mechanism reflects in vivo lung cancer patient conditions, we isolated EVs from the plasma of lung cancer patients (EVs-Patients) and heavy smokers (EVs-Donors), and we investigated their functional effects on HBEC-KRAS ${ }^{\text {V12high }}$ cells. NanoSight analysis revealed that the EVs-Patients and EVs-Donors had a similar median size (166.9 and 174.7 
A

A $\quad$-Myc (FACS)

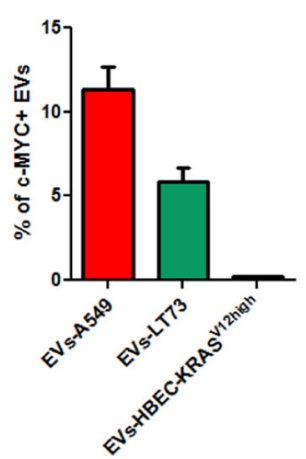

C Cell viability

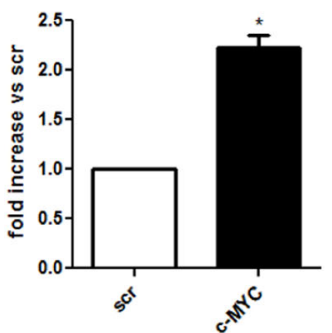

C-Myc (ELISA)

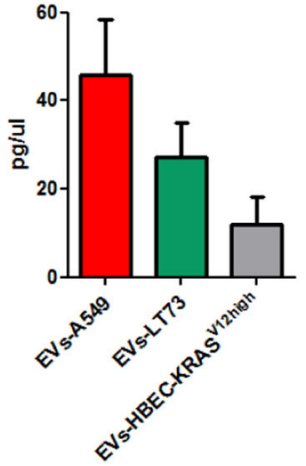

CFSE proliferation

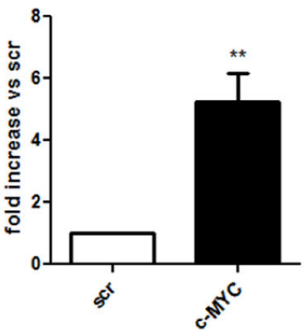

E Cell cycle analysis

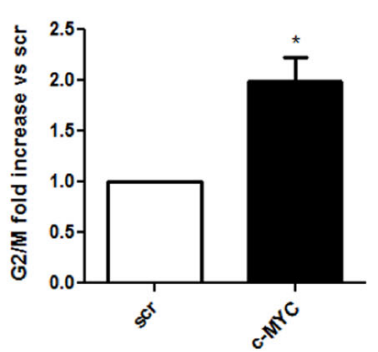

G

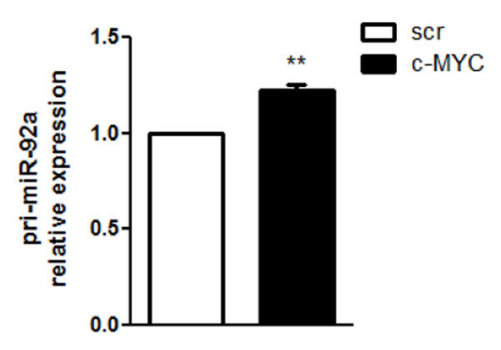

B

c-Myc (ELISA)

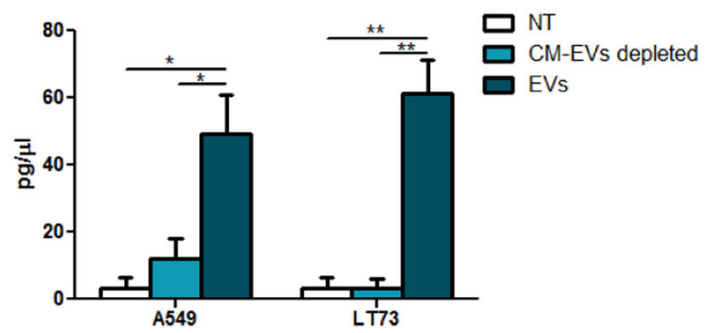

D
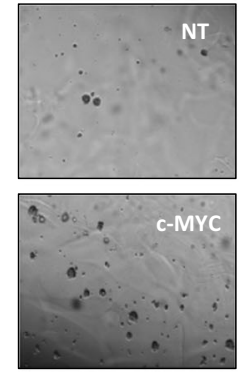

3D proliferation

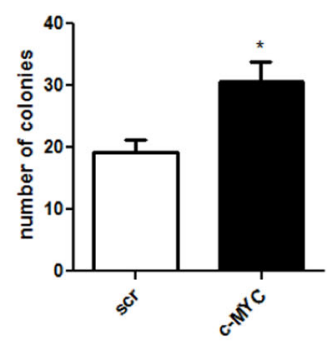

F

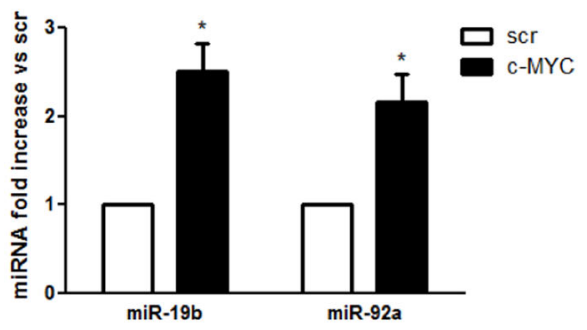

H

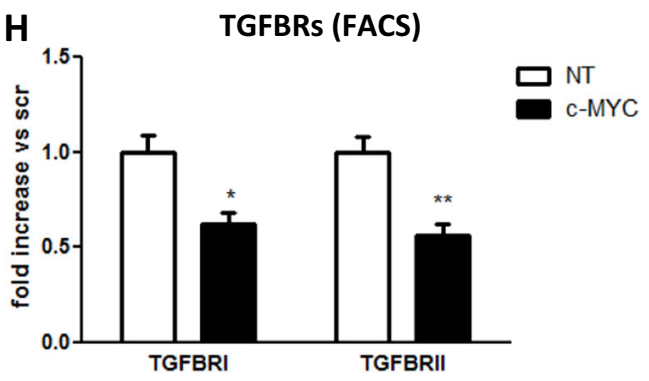

Fig. 5 c-Myc shuttled by tumour-derived EVs induced malignant transformation in recipient epithelial cells. a c-Myc protein quantification in EVs. Left: percentage of EVs-A549 (red bar), EV-LT73 (green bar) and EVs-HBEC-KRAS V12high (grey bar) positive for c-Myc expression (FACS analysis, $n=$

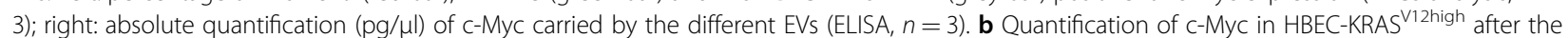
indicated treatment (ELISA, $n=3)$. c-f Graphs show cell viability (c, left; $n=3)$, CFSE (c, right; $n=3)$, 3D proliferation (d, $n=3)$ G2/M phase cells $(\mathbf{e} ; n=3)$ and TGFBRI and TGFBRII expression level $(\mathbf{f}, n=3)$ upon transient transfection of c-Myc plasmid. Data are expressed as mean \pm SEM. ${ }^{*} p<$ $0.05,{ }^{* *} p<0.01$ 
respectively, Fig. 6a, Supplementary Table 2), with greater size heterogeneity seen in the EVs-Patients (Fig. 6a). Both the EVs-Donors and EVs-Patients were positive for the exosomal markers CD9, CD63, and CD81 (Fig. 6b). The same amount $(15 \mu \mathrm{g})$ of EVs-Patients and EVs-Donors was added to HBEC-KRAS ${ }^{\text {V12high }}$ cells, and we observed an increase in the proliferation rate of the cells treated with the EVs-Patients (2-fold increase, $p<0.05$ ) that was not detectable in the cells treated with the EVs-Donors (Fig. 6c, left). CFSE proliferation (Fig. 6c, right, Supplementary Fig. 15), 3D cell culture (Fig. 6d) and cell cycle analyses (Fig. 6e) confirmed these data. Furthermore, we detected downmodulation of both TGFBRI and TGFBRII expression after treatment with EVs-Patients compared to treatment with EVs-Donors (Fig. 6f, Supplementary Figs. 11 and 12). Only the EVs derived from patients contained c-Myc protein, although the percentage varied among individuals (Fig. 6g, Supplementary Fig. 13a). This result suggests that c-Myc is specifically carried by tumoural EVs, while it is absent in EVs derived from heavy-smoker donors. As expected from this result, EVs-Patients but not EVs-Donors induced upmodulation of miR-19b and miR92a expression in recipient cells (Fig. 6h) as a consequence of the increased transcription of pri-miR-92a (Fig. 6i).

Overall, these data suggest that EVs derived from lung cancer patients can influence the tumour microenvironment and promote epithelial cell proliferation.

\section{Discussion}

It is known that lung cancer patients have an increased risk for a second primary tumour in the lung $(0.7-15 \%$ of NSCLC patients) ${ }^{37}$ or intrapulmonary metastasis (4\% in resected NSCLC) ${ }^{38}$. Our work demonstrated that EVs were able to increase proliferating activity of preneoplastic epithelial cells such as HBEC-KRASV12high which could favour tumour growth or modulate lung microenvironment for the establishment of intrapulmonary metastatic niche.

The tumour microenvironment plays a central role in cancer development and progression ${ }^{39,40}$, and in particular, EVs seem to have a crucial role in modulating the tumour microenvironment that directly surrounds the primary tumour or metastatic lesion. EVs affect several key cellular mechanisms, including oncogenic transfer, angiogenesis and pre-metastatic niche formation ${ }^{41}$. In addition, EVs are able to influence multiple aspects of the immune system, such as immunomodulation and cancer immune evasion ${ }^{42}$.

Cancer-derived EVs affect not only cells in the microenvironment but also other tumour or pre-neoplastic cells, resulting in modulation of the tumourigenic potential of the affected cells ${ }^{43,44}$. We demonstrated that EVs derived from two different lung adenocarcinoma cell lines were able to increase the proliferative ability of nontumourigenic bronchial epithelial cells. Similar to other works in which EVs were shown to modulate the malignant phenotype of epithelial cells ${ }^{17}$, our study showed increases in the proliferation and 3D growth of HBECs after treatment with lung cancer-derived EVs. This protumourigenic modulation was confirmed by the enhancement of the proportion of cells in the G2/M cell cycle phase.

The role of EV-mediated activation of recipient cells through the transfer of molecules such as proteins and miRNAs has also been described by recent studies. In line with the findings of studies that indicated the involvement of miRNAs in malignant cell transformation ${ }^{45}$, our data suggest that the modulation of epithelial cell proliferation is mediated by the upregulation of miR-19b and miR-92a transcription in HBECs. These two miRNAs are members of the miR-17/92 cluster, which is often deregulated in lung cancer and is an important regulator of the cell cycle, proliferation and other cellular processes. Interestingly, cluster members have also been described to cooperate in cell cycle control, particularly in the context of TGFB signalling also in neuroblastoma ${ }^{29,46}$. Moreover, TGFB signalling is deregulated in tumours and influences several processes, such as cell growth, differentiation, apoptosis, motility and immunity ${ }^{47,48}$. We demonstrated that lung cancer EVs increased miR-19b and miR-92a transcription, downmodulating the expression of TGFBRI and TGFBRII in treated HBECs. TGFB receptors are considered tumour suppressor genes in several cancers, and downmodulation of the expression of these receptors promotes carcinogenesis in epithelial tumours ${ }^{49,50}$.

Since we observed an increase in pri-miR-92a mRNA after EV treatment, we hypothesised that there is a transcription factor within EVs responsible for the miR-19b and miR-92a upregulation. The miR-17-92 cluster has been reported to be upregulated by the oncogene $\mathrm{c}-\mathrm{Myc}^{36}$, which can directly bind to the promoter of the miR-17/92 cluster to initiate transcription. Lobb et al. revealed the presence of this transcription factor in lung cancer exosomes ${ }^{51}$, suggesting the potential for c-Myc to be transferred from exosomes to HBECs to increase cell proliferation. Moreover, Sato et $\mathrm{al}^{28}$ found that the overexpression of c-Myc greatly enhanced malignancy in HBEC-sh-p53 + KRAS ${ }^{\text {V12 }}$ cells and induced EMT and in vivo tumour growth. Our data demonstrate how EVs secreted by tumour cells can modify the epithelial tumour microenvironment through the transfer of molecules to modulate the malignant phenotype of normal epithelial cells, with particular associations with proliferation and TGFB signalling regulation.

We clearly demonstrated that the pro-tumourigenic effects of EVs isolated from lung cancer cell lines were also observed using lung cancer patient-derived EVs, 
A
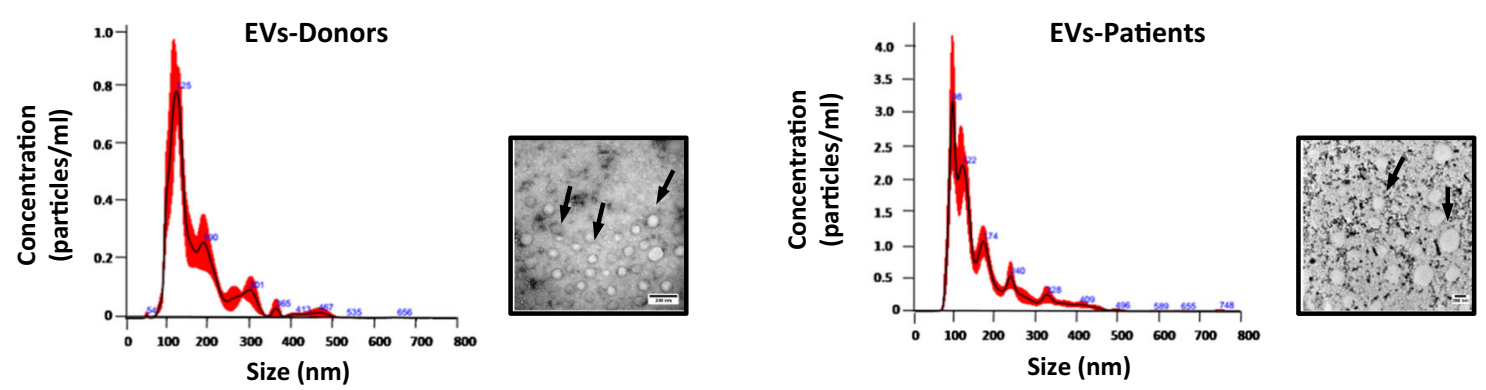

B

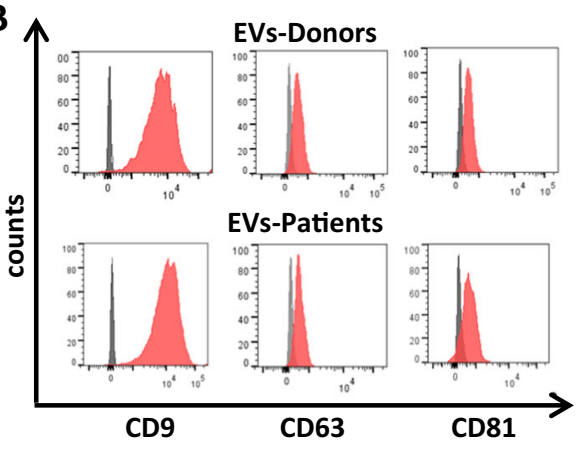

D

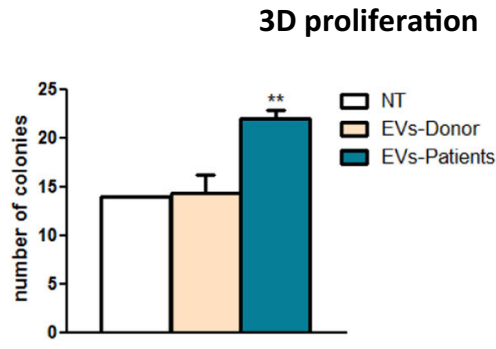

$\mathbf{F}$

TGFBRs (FACS)

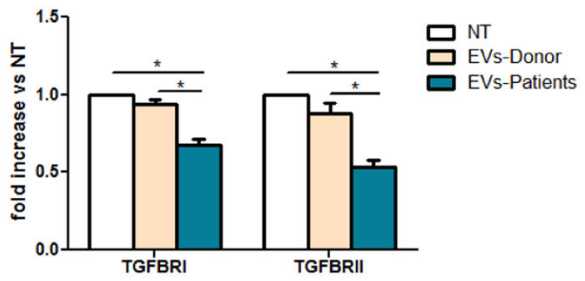

H

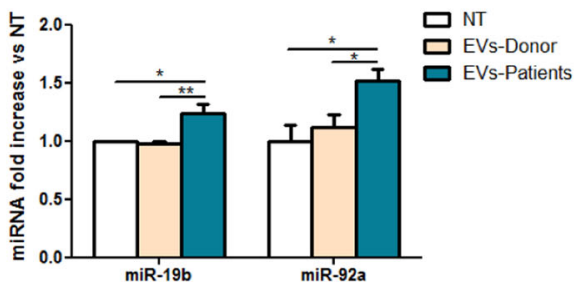

C

Cell viability

CFSE proliferation

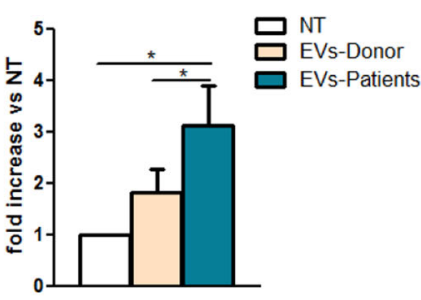

E Cell cycle analysis

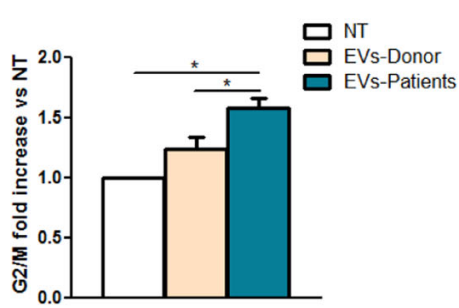

c-Myc (FACS)

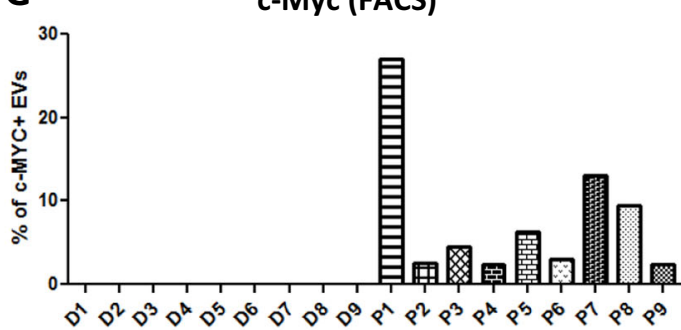

I

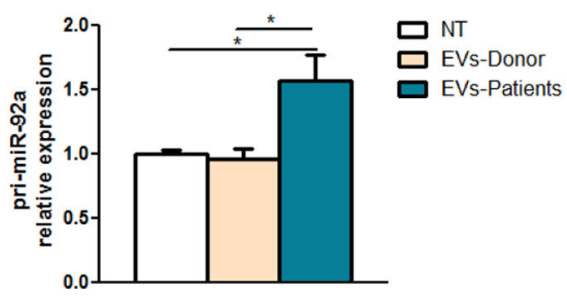

Fig. 6 (See legend on next page.) 
(see figure on previous page)

Fig. 6 Lung cancer patient EVs increased bronchial epithelial cell proliferation. a Concentration and size distribution graphs (NanoSight) and corresponding representative images (TEM) of EVs-Donors (left) and EVs-Patients (right). b FACS analysis of the exosomal markers CD9, CD63, CD81 and Alix (representative images, $n=3$ ). c-e Modulation of the HBEC-KRAS ${ }^{\text {V12high }}$ cell phenotype after EV treatment. c Cell viability (left; $\left.n=10\right)$ and CFSE proliferation (right; $n=10$ ) in cells treated with EVs-Donors and EVs-Patients compared to control-treated cells. $\mathbf{d}$ Effect of EVs on HBECKRAS $^{\text {V12high }}$ cell colony formation ability ( $n=5$; Left: quantification of colonies, right: representative images) and on the cell cycle $(\mathbf{e} ; n=6) . \mathbf{f}$ TGFBRI and TGFBRIl protein expression levels in HBEC-KRAS V12high cells treated as previously described $(n=8)$. $\mathbf{g}$ Graph bars showing the percentages of EVs positive for c-Myc in each sample (EVs derived from 9 donors (D) and 9 patients (P) using FACS). h, i miR-19b, miR-92a (h) and pri-miR-92a (i) expression levels in HBEC-KRAS ${ }^{\mathrm{V} 12 \text { high }}$ cells after EV treatment compared to no treatment $(n=10)$. Data are expressed as the mean \pm SEM. ${ }^{*} p<0.05$, ${ }^{* *} p<0.01$

mimicking what could happen in the lung cancer microenvironment in patients.

Interestingly, for the first time, we detected the presence of c-Myc inside EVs isolated from lung cancer cells and EVs isolated from the plasma of lung cancer patients. We showed that these EVs have the ability to induce premalignant changes in recipient HBEC-KRAS ${ }^{\mathrm{V} 12 \mathrm{high}}$ cells, suggesting a potential EV-mediated pro-tumourigenic mechanism that could occur in lung cancer patients.

However, whether the effect of EVs on lung epithelial cells mediated through miRNA modulation is a process limited to lung cancer or is a broader mechanism has yet to be unveiled. Our results set the basis for further analysis of the role of EVs in the plasma as biomarkers or as therapeutic tools in lung cancer.

\section{Materials and methods}

\section{Cell lines and treatments}

The human lung adenocarcinoma cell line A549 was purchased from the American Type Culture Collection (ATCC; LGC Standards), whereas the LT73 cell line was derived in our laboratory from a primary lung tumour in a 68-year-old male. Both cell lines were cultured in RPMI 1640 medium (Gibco, Thermo Fisher Scientific, Waltham, MA, USA) supplemented with $10 \%$ foetal bovine serum (FBS; EuroClone, Italy) and 1\% penicillin-streptomycin (Sigma-Aldrich, Saint Louis, MO, USA).

Immortalised bronchial epithelial cells with different genetic alterations (HBEC-KRAS ${ }^{\text {V12high }}$ cells: hTERT + Cdk $4+$ sh-p $53+$ KRAS $^{\text {V12high }}$ ) were provided by Prof. J. D. Minna ${ }^{28}$ at the University of Texas Southwestern Medical Center. HBECs were maintained in K-SFM medium (Thermo Fisher Scientific) supplemented with $5 \mathrm{ng} / \mathrm{mL}$ human recombinant EGF and $50 \mu \mathrm{g} / \mathrm{mL}$ bovine pituitary extract (Thermo Fisher Scientific). For EV isolation, complete medium was replaced with serum-free medium $48 \mathrm{~h}$ prior to isolation. Cell lines were cultured in a humidified incubator containing $5 \% \mathrm{CO}_{2}$ at $37^{\circ} \mathrm{C}$. All cell lines were authenticated by DNA short tandem repeat (STR) profiling and confirmed to be mycoplasma negative.

In total, $5 \times 10^{4} \mathrm{HBEC}-\mathrm{KRAS} \mathrm{V}^{\mathrm{V} 2 \mathrm{high}}$ cells were seeded in a 6-well plate and treated with tumour-derived EVs
$(15 \mu \mathrm{g} /$ well) or transfected with miR-19b-3p (miR-19b) or miR-92a-3p (miR-92a) mimics or a negative control (scr) $(5 \mathrm{nM}$, Thermo Fisher Scientific) using Lipofectamine 2000 (Thermo Fisher Scientific) following the manufacturer's protocols. Transfection of the appropriate LNA inhibitor (50 nM; Exiqon, Vedbæk, Denmark) was performed $24 \mathrm{~h}$ before EV or EV-depleted CM treatment (indicated as LNA EVs and LNA CM-EVs depleted, respectively), as described above. A human MYC plasmid construct was purchased from OriGene (SC112715, pCMV6-XL5-MYC; OriGene Technologies, Rockville, MD, USA) and transiently transfected into HBECKRAS ${ }^{\text {V12high }}$ cells following the company's protocol. Cells were collected at 24 or $72 \mathrm{~h}$ after treatment.

\section{Clinical specimens}

Plasma samples were collected from high-risk heavysmoker volunteers aged 50 to 75 years old, including current or former smokers with a minimum pack/year index of 30 enroled in a low-dose computed tomography (LDCT) screening trial performed at our institution (BioMild Trial) $^{52}$, and from lung cancer patients in the Istituto Nazionale Tumori (INT)-Thoracic Unit (Supplementary Table 1). Specimen collection was approved by the Internal Review and the Ethics Boards of the INT of Milan. All patients provided informed consent.

\section{$\mathrm{EV}$ isolation}

EVs from CM and plasma were purified by differential centrifugation processes, as shown in Supplementary Fig. 16. For CM-derived EV isolation, $1 \times 10^{6}$ cells $/ \mathrm{ml}$ were cultured in $175-\mathrm{cm}^{2}$ flasks for $48 \mathrm{~h}$ with serum-free medium. CM was collected and centrifuged at $300 \mathrm{x}$ g for $10 \mathrm{~min}$ and then at $3200 \times g$ for $25 \mathrm{~min}$ to remove residual cells and debris. To exclude large vesicles, the supernatant was filtered through 0.22- $\mu \mathrm{m}$ filters (Millipore, Burlington, MA, USA) and then ultracentrifuged at $120,000 \times g$ for $90 \mathrm{~min}$ at $4{ }^{\circ} \mathrm{C}$ using a TLA-100.3 fixed-angle rotor in a TL-100 ultracentrifuge (Beckman Coulter, Brea, CA, USA). The resulting supernatant was collected and stored at $-80^{\circ} \mathrm{C}$ as CM-EV depleted while the EV-enriched pellet was washed in phosphate-buffered saline (PBS; Thermo Fisher Scientific) at the same ultracentrifuge 
speed for $60 \mathrm{~min}$ at $4{ }^{\circ} \mathrm{C}$. Then the pellet was resuspended in PBS or directly lysed in RIPA buffer (Sigma-Aldrich) with protease and phosphatase inhibitors and stored at $-80^{\circ} \mathrm{C}$. The protein content of the purified EVs was determined by the Bradford assay. Regarding plasmaderived EVs, plasma was separated from whole blood as described in Fortunato et al. ${ }^{53}$. EV isolation was performed by ultracentrifugation starting with $1 \mathrm{ml}$ of stored plasma, as described above and shown in Supplementary Fig. 16.

The EV concentration and size distribution were determined by using a NanoSight NS300 instrument (Malvern Panalytical). Five 30-s videos were recorded for each sample with a camera level set at $15 / 16$ and a detection threshold set between 2 and 7 . The videos were subsequently analysed with NTA 3.2 software to calculate the size and concentration of the particles. Auto settings were used for the analysis.

\section{TEM}

EV morphology was measured using a Zeiss LIBRA 200FE transmission electron microscope with an incolumn second-generation omega filter. Samples were prepared as follows: a suspension drop $(7 \mu \mathrm{l})$ was placed on a TEM copper grid covered with a carbon/formvard film. After blotting, a negative staining procedure was performed using UranyLess (EMS-Electron Microscopy Science), a contrast agent ${ }^{54}$. The estimation of EV size was performed by measuring a hundred EVs using the iTEMTEM Imaging platform (Olympus).

\section{Western blotting}

Cells and EV pellets were lysed in RIPA buffer. Then, $40 \mu \mathrm{g}$ of protein lysate was loaded on a Bolt $4-12 \%$ BisTris gel (Thermo Fisher Scientific). Western blot analyses were performed using the following antibodies: anti-CD9 (Cell Signaling; 1:1000), anti-CD81 (Thermo Fisher Scientific; 1:100) and anti-Alix (BioLegend; 1:1000), c-Myc (Cell Signaling, 1:1000) primary antibodies and the corresponding anti-mouse and anti-rabbit peroxidase-linked secondary antibodies (GE Healthcare Life Sciences, 1:2000). Signal detection was performed via chemiluminescence reaction (ECL, GE Healthcare) using the MINI HD9 Western Blot Imaging System (Cleaver Scientific Ltd., United Kingdom). Western blot quantification was performed using ImageJ software analysis.

\section{Flow cytometry analysis}

Flow cytometry analysis of EVs was performed as previously described ${ }^{55}$, starting with $30 \mu \mathrm{g}$ of EVs. Briefly, we used $1 \mu \mathrm{g}$ each of primary anti-CD9, anti-CD81, antiCD63 (Abcam, Cambridge, UK), and anti-c-Myc (Cell Signaling, Danvers, Massachusetts, USA) antibodies and the corresponding fluorescent secondary antibodies
(Alexa Fluor 488-conjugated goat anti-rabbit IgG, Thermo Fisher Scientific; Dylight 488-conjugated goat anti-mouse IgG, Bethyl), both incubated for $30 \mathrm{~min}$ at $4{ }^{\circ} \mathrm{C}$. For c-Myc analysis, EVs were permeabilized with a $0.1 \%$ Triton solution ( $15 \mathrm{~min}$, room temperature (RT)) prior to incubation with a primary Ab.

TGFBRI analysis was performed with a primary antihTGFBRI antibody (Abcam; 1:100) and secondary Alexa Fluor 488-conjugated goat anti-rabbit antibody (Thermo Fisher Scientific; 1:2000) incubated for $1 \mathrm{~h}$ at $4{ }^{\circ} \mathrm{C}$. For TGFBRII evaluation, we used a FITC-conjugated antihTGFBRII antibody (R\&D Systems, Minneapolis, MN, USA; 1:20) incubated for $15 \mathrm{~min}$ at RT.

All analyses were performed by flow cytometry (FACSCalibur, BD Biosciences, San Jose, CA, USA) and used FlowJo software (TreeStar, Ashland, OR, USA). For c-Myc analysis, we fixed the threshold of positivity at $1 \%$ : samples with the percentage of $\mathrm{c}-\mathrm{MYC}^{+}$-exosomes below $1 \%$ were classified as negative.

\section{Enzyme-linked immunosorbent assay}

c-Myc (total) human ELISA kit (ThermoFisher Scientific) was used to measure the level of c-Myc in cellular and EVs-derived lysates, whereas RayBio Human c-Myc (MYC) ELISA Kit for cell culture supernatant samples. All tests were performed in triplicate. Absorbance at $450 \mathrm{~nm}$ was measured using a microplate reader (Infinite M1000, Tecan).

\section{miRNA and mRNA expression analyses}

MiRNA levels were determined using chip-based digital PCR (dPCR) (Thermo Fisher Scientific) as previously described $^{56}$. Briefly, total RNA was extracted using the Maxwell RSC Instrument (Promega, Madison, WI, USA) and Maxwell RSC miRNA Tissue Kit (Promega) following the manufacturer's instructions. Synthetic Caenorhabditis elegans miRNA-39 (cel-miR-39) was used as a spiked-in control and was added to each sample at a concentration of $25 \mathrm{fmol}$ from the stock tube (Qiagen, Hilden, Germany $)^{57}$. Total RNA $(20 \mathrm{ng}$ ) was reverse transcribed into cDNA using an RT primer pool specific for the miRNAs of interest. A pre-amplification step was performed using $2.5 \mu \mathrm{l}$ of $\mathrm{RT}$ product and a Custom TaqMan PreAmp primer pool (Thermo Fisher Scientific). MiRNA expression was evaluated in $2.5 \mu \mathrm{l}$ of PreAmp product using dPCR.

For analysis of EV miRNA content, $15 \mu \mathrm{g}$ of EVs were treated with RNAse A $(10 \mu \mathrm{g} / \mathrm{ml}$; Roche, Basel, Switzerland) for $30 \mathrm{~min}$ to eliminate miRNAs present outside the exosomes. Then, total RNA was extracted and analysed as described above.

Gene expression analysis was assessed by reverse transcription starting with $250 \mathrm{ng}$ of total RNA. TaqMan Gene Expression Assays (Thermo Fisher Scientific) and 
the Applied Biosystems 7900 System (Thermo Fisher Scientific) were utilised to quantify and analyse the levels of selected genes. The B2M gene was used as a reference for sample normalisation.

\section{EV labelling and internalisation}

EVs were labelled with PKH67 Fluorescent Cell Linker kits (Sigma-Aldrich) as follows: EV pellets were incubated with $50 \mu \mathrm{l}$ of PKH67 dye diluted in Diluent C (SigmaAldrich; 1:1000) for $5 \mathrm{~min}$ at RT. To eliminate excess dye, the pellet was washed with $1 \mathrm{ml}$ of PBS, ultracentrifuged at $120,000 \times g$ for $60 \mathrm{~min}$ at $4{ }^{\circ} \mathrm{C}$ and then resuspended in PBS.

For confocal microscopy analysis, PKH67-labelled EVs were added to $5 \times 10^{4}$ HBEC-KRAS ${ }^{\text {V12high }}$ cells grown on a coverslip. After $24 \mathrm{~h}$, the cells were fixed for $15 \mathrm{~min}$ in a flow cytometry fixation buffer (1X; R\&D Systems) and washed with PBS. Then, the cell membranes were stained with Alexa Fluor 633-conjugated wheat germ agglutinin (WGA) (1:2000, $1 \mathrm{mg} / \mathrm{ml}$ stock solution; Thermo Fisher Scientific) for $10 \mathrm{~min}$ at RT. The nuclei were stained with DAPI (1:1,500, $5 \mathrm{mg} / \mathrm{ml}$ stock solution; Life Technologies) for $10 \mathrm{~min}$ at RT, and the samples were mounted with ProLong Gold Antifade reagent (Thermo Fisher Scientific). A confocal laser scanning microscope (Leica TCS SP8 X) was used to visualise the samples, and Leica LAS X rel. 2.0.1 software (Leica Microsystems, Mannheim, Germany) was used to acquire images. Each fluorophore was excited independently, and sequential detection was performed. Each image consisted of a $\mathrm{z}$-series of images (step size, $0.5 \mu \mathrm{m}$ ) with $512 \times 512$ pixel resolution and was reported as extended depth of field using an HC PL APO $63 \mathrm{X} / 1.40 \mathrm{CS} 2$ oil immersion objective and a pinhole set to 1 Airy unit. The obtained data were analysed using ImageJ software.

For FACS analysis, HBEC-KRAS ${ }^{\text {12high }}$ cells were labelled with PKH26 dye (Sigma-Aldrich) according to the manufacturer's instructions and cultured with PKH67labelled EVs. In brief, the HBEC-KRAS ${ }^{\text {V12high }}$ cells were resuspended in $1 \mathrm{ml}$ of Diluent $\mathrm{C}$, and $4 \mu \mathrm{l}$ of PKH26 dye was diluted in $1 \mathrm{ml}$ of Diluent $C$. The solutions were mixed and incubated for $5 \mathrm{~min}$, with the subsequent addition of $2 \mathrm{ml}$ of FBS to bind any excess PKH26 dye. The labelled HBEC-KRAS ${ }^{\mathrm{V} 12 \text { high }}$ cells were centrifuged and then seeded in a 6 -well plate at $5 \times 10^{4}$ cells/well. The following day, the cells were incubated with the PKH67labelled EVs. All the analyses were performed using a FACSCalibur and FlowJo software.

\section{Functional assays}

Viable cells were counted after $72 \mathrm{~h}$ of treatment using a Trypan blue solution (Sigma-Aldrich). Proliferation was evaluated with a CFSE assay (Thermo Fisher Scientific) following the manufacturer's protocol.
For cell cycle analysis, HBEC-KRAS ${ }^{\text {V12high }}$ cells were harvested, washed with ice-cold PBS and fixed overnight with $70 \%$ ethanol at $4{ }^{\circ} \mathrm{C}$. After being washed twice with ice-cold PBS, the cells were incubated with a propidium iodide (PI) solution $(50 \mu \mathrm{g} / \mathrm{ml}, 0.1 \%$ Triton X100 and $0.1 \%$ sodium citrate) containing RNAse A $(0.5 \mathrm{mg} / \mathrm{ml})$ (Roche) at $37^{\circ} \mathrm{C}$ for $40 \mathrm{~min}$ in the dark. The stained cells were analysed by a FACSCalibur and FlowJo software.

A 3D proliferation assay was performed using VitroGel 3D (The well Bioscience, USA) following the manufacturer's instructions. Briefly, a 1:1 VitroGel mix was added into 24-well plates. In total, $5 \times 10^{4}$ HBECKRAS $^{\text {V12high }}$ cells were plated on top of the VitroGel, and colonies were counted after $72 \mathrm{~h}$ of treatment. Five random fields were counted for each condition.

Migration was evaluated by a wound healing assay using the JuLI Stage Instrument (NanoEnTek Inc.) and a JuLI Stage Real-Time Cell History Recorder tool.

To confirm the specific role of EVs in functional studies and exclude the potential involvement of other factors released by cells into $\mathrm{CM}$, we used treatment with EVdepleted $\mathrm{CM}$ as a negative control. Among the tested dilutions, we observed that the 1:4 dilution in K-SFM medium was useful for meeting the mentioned criteria (Supplementary Fig. 17). Untreated cells were used as an additional control.

\section{miRNA target prediction}

Computational miRNA target prediction analysis was performed using the DIANA tool and starBase v3.0 platform. In detail, DIANA-mirPath v3 and miRTarPathwaystarBase were used to identify molecular pathways potentially altered by miRNAs, while DIANA-TarBase v8 and the PITA programme were used for gene enrichment analysis of experimentally validated miRNA target genes.

The sequences of miR-19b-3p and miR-92a-3p were extracted from the miRBase database (miR-19b-3p miRBase ID: MIMAT0000074; miR-92a-3p miRBase ID: MIMAT0000092).

\section{Luciferase reporter assays}

The pLightSwitch ${ }^{\mathrm{TM}} 3^{\prime}$ UTR Reporter vector containing the 3'UTR of TGFBRII was purchased from Active Motif (ID S811544; Active Motif, Carlsbad, CA USA). A 3'UTR fragment $(468 \mathrm{bp})$ from TGBRI containing the predicted target site for miR-92a was PCR amplified from cDNA produced from HBEC-KRAS ${ }^{\text {V12high }}$ cells using the following primers:

$$
\text { Fw 5'-GGGGCTAGCAACTCTGCTGTGCTGGAGA }
$$

$T C-3^{\prime}$

Rev 5'-GGGCTCGAGCAAAACAGAAAAAGTTTGGG TTACCC-3'

and cloned into the pLightSwitchTM vector (ID S890005, Active Motif) between the NheI and XhoI 
restriction sites. Correct insertion of the TGFBRI 3'UTR was confirmed by sequencing analysis (Eurofins Genomics, Ebersberg, Germany). Predicted target sites for miR$19 \mathrm{~b}$ and miR-92a were mutated by direct mutagenesis of the pLightSwitch $3^{\prime} \mathrm{UTR}$ vectors, using the PCR-based QuikChange II site-directed mutagenesis kit (Agilent Technologies, CA, USA) according to the manufacturer's instructions and the following primers:

TGFBRI-3'UTR-Fw 5'-GGAACATAATTCATAAGGCT

GTATTTTGTATAC-3'

TGFBRI-3'UTR-Rev 5'-GTATACAAAATACAGCCTT

ATGAATTATGTTCC-3'

TGFBRII-3'UTR-Fw 5'-CAATAGCCAATAACAGGGT

TACTTTATTAATGCC-3'

TGFBRII-3'UTR-Rev5'-GGCATTAATAAAGTAACCC

TGTTATTGGCTATTG-3'

The presence of the mutations was confirmed by sequencing (Eurofins Genomics). The luciferase constructs were transfected into HEK293 cells together with miR-19b, miR-92a or a scrambled oligonucleotide sequence. The cells were cultured for $48 \mathrm{~h}$ and assayed with the Luciferase Reporter Assay System (Active Motif, Carlsbad, CA USA) according to the manufacturer's instructions.

\section{Statistical analyses}

All experiments were performed at least in triplicate, and all values are reported as the mean \pm SEM. Analyses were performed using GraphPad Prism (GraphPad Software, La Jolla, California USA). Intergroup comparisons were assessed by a two-tailed Student's $t$-test, and a $p$-value $<0.05$ was considered statistically significant. Statistical significance has been indicated as follows: ${ }^{*} p<$ $0.05, " * x<0.01$, and $* * * * 0.001$.

\section{Acknowledgements}

The study was supported by grants from the Italian Association for Cancer Research [Investigator Grant Nos. 14318 and 18812 to G.S., and 12162 G.S. (Special Program "Innovative Tools for Cancer Risk Assessment and early Diagnosis", $5 \times 1000)$ ); the Italian Ministry of Health [Grant No. RF-2010] and $\mathrm{NIH/U01CA166905}$. O.F. was supported by the Cariplo Foundation Young Investigator Grant 2015 n.2015-0901. We thank Viviana Bornaghi for providing technical assistance.

\section{Author details}

${ }^{1}$ Tumor Genomics Unit, Department of Research, Fondazione IRCCS Istituto Nazionale dei Tumori, 20133 Milan, Italy. ${ }^{2}$ Lab. Di nanotecnologie, Istituto di scienze e tecnologie Molecolari CNR, 20138 Milan, Italy. ${ }^{3}$ Unit of Molecular Bases of Genetic Risk and Genetic Testing, Fondazione IRCCS Istituto Nazionale dei Tumori, 20133 Milan, Italy. ${ }^{4}$ Thoracic Surgery Unit, Fondazione IRCCS Istituto Nazionale dei Tumori, 20133 Milan, Italy

\section{Conflict of interest}

The authors declare that they have no conflict of interest.

\section{Publisher's note}

Springer Nature remains neutral with regard to jurisdictional claims in published maps and institutional affiliations.
Supplementary Information accompanies this paper at (https://doi.org/ 10.1038/s41419-019-2003-5).

Received: 17 April 2019 Revised: 13 September 2019 Accepted: 23 September 2019

Published online: 07 October 2019

\section{References}

1. Malvezzi, M. et al. European cancer mortality predictions for the year 2018 with focus on colorectal cancer. Ann. Oncol. 29, 1016-1022 (2018).

2. Herbst, R. S., Morgensztern, D. \& Boshoff, C. The biology and management of non-small cell lung cancer. Nature 553, 446-454 (2018).

3. Siegel, R. L., Miller, K. D. \& Jemal, A. Cancer statistics, 2018. CA Cancer J. Clin. 68, 7-30 (2018).

4. Postmus, P. E. et al. Early and locally advanced non-small-cell lung cancer (NSCLC): ESMO Clinical Practice Guidelines for diagnosis, treatment and follow-up. Ann. Oncol. 28, iv1-iv21 (2017).

5. Carbone, D. P., Gandara, D. R., Antonia, S. J., Zielinski, C. \& Paz-Ares, L. Nonsmall-cell lung cancer: role of the immune system and potential for immunotherapy. J. Thorac. Oncol. 10, 974-984 (2015).

6. Bremnes, R. M. et al. The role of tumor stroma in cancer progression and prognosis: emphasis on carcinoma-associated fibroblasts and non-small cell lung cancer. J. Thorac. Oncol. 6, 209-217 (2011).

7. Pullamsetti, S. S. et al. Lung cancer-associated pulmonary hypertension: Role of microenvironmental inflammation based on tumor cell-immune cell crosstalk. Sci. Transl. Med. 9, eaai9048 (2017).

8. Engblom, C. et al. Osteoblasts remotely supply lung tumors with cancerpromoting SiglecF(high) neutrophils. Science 358, eaal5081 (2017).

9. Nawaz, M. \& Fatima, F. Extracellular vesicles, tunneling nanotubes, and cellular interplay: synergies and missing links. Front Mol. Biosci. 4, 50 (2017).

10. Maia, J., Caja, S., Strano Moraes, M. C., Couto, N. \& Costa-Silva, B. Exosomebased cell-cell communication in the tumor microenvironment. Front. Cell Dev. Biol. 6, 18 (2018).

11. Colombo, M., Raposo, G. \& Thery, C. Biogenesis, secretion, and intercellular interactions of exosomes and other extracellular vesicles. Annu. Rev. Cell Dev. Biol. 30, 255-289 (2014).

12. Thery, C., Zitvogel, L. \& Amigorena, S. Exosomes: composition, biogenesis and function. Nat. Rev. Immunol. 2, 569-579 (2002).

13. Fatima, F. \& Nawaz, M. Vesiculated long non-coding rnas: offshore packages deciphering trans-regulation between cells, cancer progression and resistance to therapies. Noncoding RNA 3, E10(2017).

14. Ruivo, C. F., Adem, B., Silva, M. \& Melo, S. A. The biology of cancer exosomes: insights and new perspectives. Cancer Res. 77, 6480-6488 (2017).

15. Zheng, $H$. et al. The roles of tumor-derived exosomes in non-small cell lung cancer and their clinical implications. J. Exp. Clin. Cancer Res. 37, 226 (2018).

16. Liu, Y. et al. STAT3-regulated exosomal miR-21 promotes angiogenesis and is involved in neoplastic processes of transformed human bronchial epithelial cells. Cancer Lett. 370, 125-135 (2016).

17. Rahman, M. A. et al. Lung cancer exosomes as drivers of epithelial mesenchymal transition. Oncotarget 7, 54852-54866 (2016).

18. Lobb, R. J. et al. Exosomes derived from mesenchymal non-small cell lung cancer cells promote chemoresistance. Int J. Cancer 141, 614-620 (2017).

19. Jonas, S. \& Izaurralde, E. Towards a molecular understanding of microRNAmediated gene silencing. Nat. Rev. Genet. 16, 421-433 (2015).

20. Croce, C. M. Causes and consequences of microRNA dysregulation in cancer. Nat. Rev. Genet. 10, 704-714 (2009).

21. Boeri, M. et al. MicroRNA signatures in tissues and plasma predict development and prognosis of computed tomography detected lung cancer. Proc Natl Acad. Sci. USA 108, 3713-3718 (2011).

22. Sozzi, G. et al. Clinical utility of a plasma-based miRNA signature classifier within computed tomography lung cancer screening: a correlative MILD trial study. J. Clin. Oncol. 32, 768-773 (2014).

23. Fortunato, O. et al. Mir-660 is downregulated in lung cancer patients and its replacement inhibits lung tumorigenesis by targeting MDM2-p53 interaction. Cell Death Dis. 5, e1564 (2014).

24. Borzi, C. et al. mir-660-p53-mir-486 network: a new key regulatory pathway in lung tumorigenesis. Int. J. Mol. Sci. 18, E222 (2017) 
25. Fabbri, M. et al. MicroRNAs bind to Toll-like receptors to induce prometastatic inflammatory response. Proc. Natl Acad. Sci. USA 109, E2110-E2116 (2012).

26. Hsu, Y. L. et al. Hypoxic lung cancer-secreted exosomal miR-23a increased angiogenesis and vascular permeability by targeting prolyl hydroxylase and tight junction protein ZO-1. Oncogene 36, 4929-4942 (2017).

27. Hsu, Y. L. et al. Hypoxic lung-cancer-derived extracellular vesicle microRNA$103 a$ increases the oncogenic effects of macrophages by targeting PTEN. Mol. Ther. 26, 568-581 (2018).

28. Sato, M. et al. Human lung epithelial cells progressed to malignancy through specific oncogenic manipulations. Mol. Cancer Res. 11, 638-650 (2013).

29. Mestdagh, $P$. et al. The miR-17-92 microRNA cluster regulates multiple components of the TGF-beta pathway in neuroblastoma. Mol. Cell 40, 762-773 (2010).

30. Dews, M. et al. The myc-miR-17 92 axis blunts TGF\{beta\} signaling and production of multiple TGF\{beta\}-dependent antiangiogenic factors. Cancer Res. 70, 8233-8246 (2010).

31. Jiang, Z. et al. MiRNA 17 family regulates cisplatin-resistant and metastasis by targeting TGFbetaR2 in NSCLC. PLoS One 9, e94639 (2014).

32. lavarone, A. \& Massague, J. Repression of the CDK activator Cdc25A and cellcycle arrest by cytokine TGF-beta in cells lacking the CDK inhibitor p15. Nature 387, 417-422 (1997)

33. Pardali, K., Kowanetz, M., Heldin, C. H. \& Moustakas, A. Smad pathway-specific transcriptional regulation of the cell cycle inhibitor p21(WAF1/Cip1). J. Cell Physiol. 204, 260-272 (2005).

34. Korah, J., Falah, N., Lacerte, A. \& Lebrun, J. J. A transcriptionally active pRb-E2F1P/CAF signaling pathway is central to TGFbeta-mediated apoptosis. Cell Death Dis. 3, e407 (2012)

35. Scandura, J. M., Boccuni, P., Massague, J. \& Nimer, S. D. Transforming growth factor beta-induced cell cycle arrest of human hematopoietic cells requires p57KIP2 up-regulation. Proc. Natl Acad. Sci. USA 101, 15231-15236 (2004).

36. O'Donnell, K. A., Wentzel, E. A., Zeller, K. I., Dang, C. V. \& Mendell, J. T. c-Mycregulated microRNAs modulate E2F1 expression. Nature 435, 839-843 (2005).

37. Martini, N. et al. Incidence of local recurrence and second primary tumors in resected stage I lung cancer. J. Thorac. Cardiovasc. Surg. 109, 120-129 (1995).

38. Oliaro, A. et al. The significance of intrapulmonary metastasis in non-small cell lung cancer: upstaging or downstaging? A re-appraisal for the next TNM staging system. Eur. J. Cardiothorac. Surg. 34, 438-443 (2008).

39. Witz, I. P. The tumor microenvironment: the making of a paradigm. Cancer Microenviron. 2, 9-17 (2009).
40. Joyce, J. A. \& Pollard, J. W. Microenvironmental regulation of metastasis. Nat Rev. Cancer 9, 239-252 (2009).

41. Xu, R., Greening, D. W., Zhu, H. J., Takahashi, N. \& Simpson, R. J. Extracellular vesicle isolation and characterization: toward clinical application. J. Clin. Invest. 126, 1152-1162 (2016).

42. Whiteside, T. L. Exosomes and tumor-mediated immune suppression. J. Clin. Invest. 126, 1216-1223 (2016).

43. Kosaka, N. et al. Secretory mechanisms and intercellular transfer of microRNAs in living cells. J. Biol. Chem. 285, 17442-17452 (2010).

44. Becker, A. et al. Extracellular vesicles in cancer: cell-to-cell mediators of metastasis. Cancer Cell 30, 836-848 (2016).

45. Kosaka, N., Yoshioka, Y., Fujita, Y. \& Ochiya, T. Versatile roles of extracellular vesicles in cancer. J. Clin. Invest. 126, 1163-1172 (2016).

46. Mogilyansky, E. \& Rigoutsos, I. The miR-17/92 cluster: a comprehensive update on its genomics, genetics, functions and increasingly important and numerous roles in health and disease. Cell Death Differ. 20, 1603-1614 (2013).

47. Neuzillet, C. et al. Targeting the TGFbeta pathway for cancer therapy. Pharmacol. Ther. 147, 22-31 (2015)

48. Caja, F. \& Vannucci, L. TGFbeta: a player on multiple fronts in the tumor microenvironment. J. Immunotoxicol. 12, 300-307 (2015).

49. Yang, $\mathrm{H}$. et al. Concomitant underexpression of TGFBR2 and overexpression of hTERT are associated with poor prognosis in cervical cancer. Sci. Rep. 7, 41670 (2017).

50. Massague, J. TGFbeta in ancer. Cell 134, 215-230 (2008)

51. Lobb, R. J. et al. Oncogenic transformation of lung cells results in distinct exosome protein profile similar to the cell of origin. Proteomics 17, 23-24 (2017).

52. Boeri, M. et al. Recent advances of microRNA-based molecular diagnostics to reduce false-positive lung cancer imaging. Expert Rev. Mol. Diagn. 15, 801-813 (2015).

53. Fortunato, $\mathrm{O}$. et al. Assessment of circulating microRNAs in plasma of lung cancer patients. Molecules 19, 3038-3054 (2014).

54. Placzek, M. \& Kosela, M. Microscopic methods in analysis of submicron phospholipid dispersions. Acta Pharm. 66, 1-22 (2016).

55. Thery, C., Amigorena, S., Raposo, G. \& Clayton, A. Isolation and characterization of exosomes from cell culture supernatants and biological fluids. Curr. Protoc. Cell Biol Chapter 3, Unit 3.22 (2006).

56. Conte, D. et al. Novel method to detect microRNAs using chip-based QuantStudio 3D digital PCR. BMC Genomics 16, 849 (2015).

57. Kroh, E. M., Parkin, R. K., Mitchell, P. S. \& Tewari, M. Analysis of circulating microRNA biomarkers in plasma and serum using quantitative reverse transcription-PCR (qRT-PCR). Methods 50, 298-301 (2010). 